
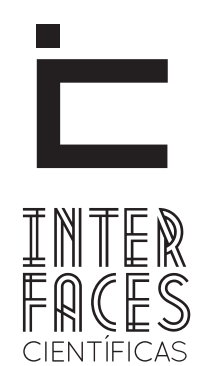

EDUCAÇÃO

ISSN IMPRESSO 2316-333X

E-ISSN 2316-3828

DOI-10.17564/2316-3828.2016v5n1p29-40

\title{
O PROGRAMA MAIS CULTURA NAS UNIVERSIDADES COMO EXEMPLO INTERSETORIAL DE POLÍTICA PÚBLICA ENTRE CULTURA E EDUCAÇÃo
}

Camila Cestari Cerreti $^{1}$
Tony Gigliotti Bezerra²

\section{RESUMO}

O presente artigo se propõe analisar a construção do Programa Mais Cultura nas Universidades, com base nas ações realizadas e perspectivas políticas do Ministério da Cultura em articulação com o Ministério da Educação até a recente instituição do referido Programa. Os autores analisam o desafio do processo de gestão desta política interministerial, partindo da complexidade do estabelecimento de estratégias e contextos de conexão e legitimação entre a sociedade civil e o ambiente acadêmico.

\section{PALAVRAS-CHAVE}

Programa Mais Cultura nas Universidades. Política Pública Cultural. Ensino Superior. Cultura. Educação. 


\section{ABSTRACT}

This article aims to analyze the construction of the "More Culture in Universities" Program, based on the actions taken and the political perspectives to the Ministry of Culture in conjunction with the Ministry of Education, until the recent institution of this Program. The author analyzes the challenge of the management process of this ministerial policy, based on the complexity of establishment the strategies and

\section{RESUMEN}

Este artículo tiene como objetivo analizar la construcción del Programa Mais Cultura nas Universidades, en función de las medidas adoptadas y de perspectivas políticas del Ministerio de Cultura en colaboración con el Ministerio de Educación en Brasil, hasta la reciente institución del Programa. La autora analiza el desafío del proceso de gestión de esta política ministerial, desde la complejidad de la creación de es- connection settings for legitimacy between civil society and the academic environment.

\section{KEYWORDS}

"More Culture in the Universities" Program. Cultural Public Policy. Higher Education. Culture. Education. trategias y ajustes de conexión y legitimidad entre la sociedad civil y el ámbito académico.

\section{PALABRAS CLAVE}

Programa Mais Cultura nas Universidades, Política Pública Cultural, Educación Superior, Cultura, Educación. 


\section{INTRODUÇ̃̃̃O}

A importância da cultura no mundo contemporâneo tem sido enfatizada por autores de diferentes tendências. No âmbito do pensamento pós-moderno, a cultura adquire e cada vez mais significativo conceitualmente, a exemplo do que afirma Edgar Morin (2000, p. 56):

A cultura é constituída pelo conjunto dos saberes, fazeres, normas, proibições, estratégias, crenças, ideias, valores, mitos, que se transmite de geração em geração, se reproduz em cada indivíduo, controla a existência da sociedade e mantém a complexidade psicológica e social.

Desse modo, em sua relação com a educação, a cultura se torna norteadora da mesma, ficando a educação a serviço da cultura, reafirmando uma ideologia, uma cultura hegemônica. Assim, ambas se influenciam, tornando-se indissociáveis. Para Isaura Botelho, a educação é o principal mecanismo de transmissão formal da cultura, sendo necessária uma maior integração entre as duas políticas públicas. (MINISTÉRIO..., 2006, p. 50).

Em um panorama breve, a relação histórica das políticas públicas de cultura é marcada pelo cenário político brasileiro e o contexto da trajetória do exercício de suas gestões, tendo iniciado dentro do Ministério da Educação e Saúde, posteriormente com a criação do Ministério da Cultura (MinC) e atualmente com a recente aproximação destas políticas em diálogo com o Ministério da Educação (MEC). José Marcio Barros, em sua palestra durante o Seminário Cultura e Universidade, elucida sinteticamente a questão central que permeia este processo:

Questão importante refere-se ao fato de que, o chamamento da Cultura ao campo da Educação, é um chamamento complexo, urgente, necessário, mas que no meu entendimento, não pode repetir as práticas desse divórcio, desse desquite que há 28 anos a separação do MinC e do MEC representou para esse país. Se o objetivo era elevar a Cultura ao lugar que ela merece, isso foi realizado, mas ao custo caríssimo, de um distanciamento estrutural com as questões da educação e da escola. (BARROS, 2013,).

0 processo de institucionalização das políticas culturais teve, entre os seus marcos históricos, a criação de uma secretaria subordinada ao MEC, durante a ditadura militar iniciada em 1964. Neta ocasião, a estratégia do governo era a de utilizar a cultura como instrumento tático de legitimação do poder controlador de sua ideologia. No ano de 1985, marco histórico da política cultural brasileira, ocorre o movimento de redemocratização do cenário político com a criação do MinC, enquanto uma pasta a serviço da instituição das políticas públicas culturais. Nos anos recentes, balizados pela realização de metas do Plano Nacional de Cultura (PNC) e pelo processo de institucionalização do Sistema Nacional de Cultura (SNC), evidencia-se a necessidade de se promover, verdadeiramente, a aproximação e integração entre as duas pastas.

A criação do programa Mais Cultura nas Universidades teve como fundamento as diretrizes que norteiam as bases da política nacional de cultura, quais sejam: concretizar o aproveitamento e aprimoramento das ações e programas que propiciem o amadurecimento do diálogo entre cultura e educação; e promover a integração entre educação e cultura com vistas a fazer da escola o grande espaço para a circulação da cultura brasileira, para o acesso aos bens culturais e para o respeito à diversidade e pluralidade da cultura nacional (Portaria Interministerial MinC/MEC $n^{0} 18$, 2013). Com este intuito, foi firmada uma parceria entre os Ministérios da Cultura e da Educação, por meio de Acordo de Cooperação Técnica 001/2011, onde assumiram o compromisso de desenvolvimento de ações conjuntas para a implementação de uma política integrada que contribuísse com o desenvolvimento nacional, a partir do desenvolvimento cultural.

Com este advento, foi criada, no primeiro semestre de 2012, a Diretoria de Educação e Comunicação para a Cultura da Secretaria de Políticas Culturais 
do MinC, que assumiu o desenvolvimento de políticas transversais, relacionando a cultura com a educação e a comunicação, de modo a promover a integração entre o Plano Nacional de Cultura (PNC), - Plano Nacional de Educação (PNE), e as políticas de comunicação. Desenvolver uma política nacional de articulação entre educação e cultura, que promova o reconhecimento das artes como campo do conhecimento e dos saberes culturais como elemento estratégico para qualificação do processo cultural e educativo constituiu-se como o desafio central deste acordo interministerial.

\section{PROGRAMA MAIS CULTURA NAS UNIVERSIDADES: OS AMBIENTES DE CONSTRUÇ̃̃O E OS DESDOBRAMENTOS DE SUA EFETIVAÇ̃̃O}

Ao delimitarmos o horizonte histórico/político deste estudo, observamos que o diálogo das políticas de educação e cultura exerce na sociedade contemporânea, um papel de relevância estratégica, evidenciando a importância da aplicação dos princípios da democracia participativa na elaboração de tais políticas, objetivando o desenvolvimento humano. Descreveremos brevemente o contexto de articulação política que precedeu o Programa Mais Cultura nas Universidades (PMCU) no âmbito nacional. Esta contextualização histórica faz-se necessária para que possamos compreender as vicissitudes da relação entre a política cultural e a participação social.

O debate acerca das Políticas Culturais nas instituições de nível superior veio galgando representatividade na contemporaneidade. É um processo crescente na produção acadêmica 0 compromisso em analisar as políticas culturais, repensando seu papel - da academia - com a responsabilidade competente de produção e circulação da cultura, buscando valorizar a produção cultural universitária e popular, podendo ver a cultura e sua importância simbólica na pesquisa, no ensino e extensão.

A investida colaborativa para legitimar essas mudanças resultou no Seminário Cultura e Universidade $^{2}$, que visou uma parceria entre as Universidades Públicas e o Ministério da Cultura com vistas à construção de políticas de cultura para o ensino superior do país. Sendo a Universidade importante vetor de desenvolvimento social, este seminário contribuiu para o início formal de um processo de legitimação das políticas na área da cultura.

Intitulado por I Seminário Cultura e Universidade - Bases para uma política nacional de cultura para as Instituições de Ensino Superior, foi realizado pela Universidade Federal da Bahia em parceria com o MinC, o MEC e o Fórum de Pró-reitores de Extensão das Universidades Públicas (FORPROEXT), com duração de 3 dias, acontecendo entre 22 a 24 de abril de 2013, em Salvador-BA.

Estiveram presentes mais de trezentas pessoas de todo o país da área acadêmica, com destaques específicos às classes representativas de pró-reitores de extensão, representantes dos estudantes e das rádios, TVs e editoras universitárias, diretores de institutos e centros de artes, gestores de órgãos públicos, relacionados com pesquisa, educação e cultura, como MinC, MEC, Ministério da Ciência, Tecnologia e Inovação (MCTI), Comissão de Aperfeiçoamento de Pessoal do Nível Superior (CAPES), Conselho Nacional de Pesquisa (CNPq), Financiadora de Estudos e Projetos (FINEP), assim como também representantes da sociedade civil e dos movimentos que possuem inserção em temas que envolvem o desenvolvimento de políticas públicas de educação e cultura.

2. Este Seminário teve título e I Seminário Cultura e Universidade, mas em 2003, na gestão de Gilberto Gil, o MinC já havia realizado, em Salvador-BA, um Seminário nesta temática, porém este não obteve registro. Em respeito à denominação do Ministério da Cultura será mantido no corpo do texto a enumeração do referido seminário como "I Seminário Cultura e Universidade". 
O Seminário objetivou sensibilizar, mobilizar e articular Instituições Públicas de Ensino Superior para dialogar sobre ações que provessem o desenvolvimento da cultura. Foram promovidas diversas formas de troca, levando em consideração a riqueza dessa variedade de instâncias da sociedade, reunidas para refletir sobre o desenvolvimento da cultura por meio de programas que contemplassem os cursos de formação, pesquisa e extensão em arte e cultura.

A Coordenação Nacional de Cultura do Fórum de Pró-Reitores de Extensão (FORPROEX) foi a maior responsável pela mobilização do Seminário, sendo bem sucedida a intenção de articular a inteligência acadêmica coletiva a fim de formular, de maneira sistêmica, um Programa que contemplasse a diversidade de possibilidades que envolvem o tema da cultura e das artes nos campos da pesquisa, ensino e extensão. Todo este trabalho, posteriormente, resultou no PMCU. Para atingir este objetivo, foram necessários dois anos de diálogo entre diversas instituições do ensino superior para tornar possível horizontes mais amplos que engendrassem o desenvolvimento de uma parceria, que pudesse favorecer efetivamente uma política pública federal e interministerial - entre MinC e MEC. Todavia, os esforços de traçar o delineamento de um Programa deste porte não poderiam ser concebidos substancialmente pela classe de PróReitores de extensão das Universidades.

Após a realização do Seminário Cultura e Universidade, foi feita uma reivindicação por parte dos Institutos de Artes que não foram significativamente representados no evento. Diante deste contexto, a Universidade Federal de Ouro Preto, em parceria com o MinC e o FORPROEX, realizaram o / Encontro Nacional do Ensino Superior das Artes, entre os dias 24 e 26 de março de 2014, em Ouro Preto-MG, para ouvir as contribuições e demandas dos gestores de cursos de artes. Tal como o Seminário de Salvador, este encontro foi de grande importância, posto que, pela primeira vez, dirigentes dos cursos universitários de Artes se reuniram para debater as políticas públicas de cultura nas universidades, uma oportunidade ímpar para o MinC e a academia amadurecerem o diálogo e o entendimento da formação em Artes nas IES.

O principal produto deste encontro foi a Carta de Ouro Preto que minutou as demandas do setor. Os dirigentes dos cursos de formação de artes presentes no encontro legitimaram no evento este espaço de construção, chamando atenção para a:

[...] necessidade de implementação de condições adequadas para o pleno exercício da nossa sensibilidade, do nosso potencial criativo traduzido também em nossa prática docente que, por sua vez, precisa alçar voo e romper com as estruturas de valores que imobilizam, emudecem, apagam e minam nossas ações. (CARTA DE OURO PRETO, 2014).

Então puderam diagnosticar as suas necessidades se afirmando enquanto grupo político dentro da Universidade. Esta postura fica evidente quando afirmam, na mesma carta:

\begin{abstract}
Já somos capazes de estabelecer uma discussão madura sobre Arte no Brasil. Os diferentes grupos, discutindo suas especificidades, chegaram a um apontamento comum que nos impele à criação de um espaço contínuo de diálogo, avaliação e proposição de ações. Então, sem imaginar a dimensão que teria, o I Encontro Nacional do Ensino Superior das Artes, ancorado na proposição da efetivação de diálogo motivado pela publicação da Portaria Interministerial, $n^{0} 18$, de 18 de dezembro de 2013, acabou por descortinar questões que demandavam ações que há muito permaneciam conditas nas celas individuais dos nossos cotidianos. (CARTA..., 2014).
\end{abstract}

O desenvolvimento de políticas públicas de cultura para as universidades sempre foi uma demanda histórica e, com o crescimento do número de universidades, cursos e vagas, sua efetivação e maturação se acelerou. Antes do acordo interministerial de 2011, a participação do MinC no ensino superior era marcada pela descontinuidade, por meio de ações pontuais, como apoio a projetos, pesquisas acadêmicas, Seminários etc. Incentivados por meio da descentralização de recursos, com parcerias firmadas 
por convênios. Embora esta modalidade ainda seja praticada, o PMCU tem como perspectiva inaugurar um marco formal das políticas públicas da área cultural voltadas para o ensino superior, traçando perspectivas de longo prazo, compreendendo a necessidade de realizar ações que contemplem o fomento e a articulação da cultura nas universidades, estimulando a continuidade e amadurecimento das políticas culturais no ensino superior.

Fundamentado nos Arts. 205, 206, 207, 208, 214 215, 216 e 216-A da Constituição Federal (1988), que estabelece que "o Estado garantirá a todos o direito à educação e o pleno exercício dos direitos culturais e acesso às fontes da cultura nacional, apoiará e incentivará a valorização e a difusão das manifestações culturais", também, considerando o Plano Nacional de Educação, o Plano Nacional de Cultura e o Sistema Nacional de Cultura, pautado na necessidade de integração entre educação e cultura para desenvolvimento e fortalecimento do campo artístico e cultural no Brasil, o PMCU foi instituído por meio da Portaria MEC/MinC $n^{0} 18$, publicada em dezembro de 2013.

Após as discussões e articulações que alimentaram a formulação das bases e diretrizes do PMCU, apresentadas anteriormente, foi constituído um Grupo de Trabalho (GT), a Comissão Interministerial - Cultura e Educação, responsável pela construção da portaria supracitada, que instituiu o Programa e o Fórum Nacional de Formação e Inovação em Arte e Cultura, nomeado por meio da portaria interministerial $n^{0} 6$, de 29 de maio de 2014, que recebeu as propostas das Universidades para a elaboração do edital.

Tal comissão foi anunciada no caput do Art. $7^{\circ}$, da portaria interministerial $n^{0} 18$, de 18 de dezembro de 2013, que cria o "Comitê Técnico, pelos Ministérios da Educação e da Cultura, especificamente para os fins do Programa, e basear-se-á em metas, critérios de priorização e pré-requisitos fixados através de edital, previstos neste instrumento." Vale destacar os desdobramentos deste artigo, explicitando seus parágrafos, para melhor compreensão estrutural do programa:

$\S 1^{0}$ - O Comitê Técnico será composto por seis membros, sendo 2 (dois) indicados pelo MEC, 2 (dois) indicados pelo Ministério da Cultura - MinC, 1 (um) indicado pela Associação Nacional dos Dirigentes das Instituições Federais de Ensino Superior - Andifes e 1 (um) indicado pelo Conselho Nacional das Instituições da Rede Federal de Educação Profissional, Científica e Tecnológica - Conif, nomeados por portaria conjunta do MEC e do MinC.

$\S 2^{\circ}$ As instituições selecionadas, e suas parceiras, passam a integrar o Fórum Nacional de Formação e Inovação em Arte e Cultura.

$\S 3^{0}$ - O Comitê Técnico fará a seleção dos Planos de Cultura conforme estabelecido nos arts. $5^{\circ}$ e $6^{\circ}$ desta Portaria.

A importância de implantação do referido Fórum relaciona-se à necessidade de que o programa seja acompanhado e avaliado, além de se constituir como uma instância de representação, articulação e deliberação dos diferentes entes supracitados, ligados ao PMCU. O Programa define ainda que a coordenação estará a cargo de um Comitê Técnico, definido no caput do Art. $2^{\circ}$, da portaria interministerial $\mathrm{n}^{0}$ 18, de 18 de dezembro de 2013 da seguinte forma: "Fica instituído o Fórum Nacional de Formação e Inovação em Arte e Cultura, com o objetivo de articular e congregar as instituições relacionadas nos $\S \S 1^{\circ}$ e $2^{\circ}$ do art. $1^{\circ}$ desta Portaria”. No entanto, este Fórum ainda não foi implantado.

Tais instituições relativas ao parágrafo $1^{0}$ são as Universidades Federais e as Instituições da Rede Federal de Educação Profissional, Científica e Tecnológica (IFES) e, relativas ao parágrafo $2^{\circ}$, as outras instituições de caráter cultural, artístico ou educacional para as finalidades deste Programa, bem como Universidades Estaduais e Municipais que também podem ser indiretamente beneficiadas pelo Programa por meio de projetos em parceria com as IFES, questão que também analisaremos mais à frente. 
O PMCU foi criado com a finalidade de possibilitar o desenvolvimento e o fortalecimento do campo da arte e da cultura, nas suas dimensões: simbólica, cidadã e econômica, com ênfase na inclusão social e no respeito e reconhecimento da diversidade cultural, gerando condições para fortalecer seus agentes e meios de atuação e inovação nas diversas esferas e escalas do desenvolvimento territorial do país.

Os recursos para execução das atividades a serem apoiadas no PMCU estão garantidos formalmente, de acordo com o Art. 10, da portaria interministerial $n^{0} 18$, de 18 de dezembro de 2013, pelo MEC que reservará ao Programa recursos orçamentários e financeiros que serão destinados às IFES, desde que selecionadas em edital, especificamente no que concerne a:

I - Ações relacionadas ao desenvolvimento de atividades artísticas e culturais, articuladas com ensino, pesquisa, extensão nos diferentes níveis e modalidades de ensino, voltadas aos diferentes níveis de formação (básico, técnico, graduação e pós-graduação, lato e strictu sensu) e aos diferentes segmentos sociais envolvidos nas ações; e

II - aquisição de equipamentos, serviços e despesas de custeio associadas às atividades decorrentes do Programa e definidas no Plano de Cultura aprovado por edital.

A implementação da primeira fase do programa se deu ao longo de 2014 com a instalação da Comissão interministerial - Cultura e Educação e o lançamento do edital, realizado dia 8 de outubro de 2014, que estabelece a responsabilidade de uma Comissão Técnica para receber os projetos, realizar as primeiras triagens, formar a comissão de seleção etc. Ou seja, o gerenciamento massivo do programa. Toda a descentralização da verba do PMCU será oriunda dos recursos orçamentários do MEC bem como a forma de repasse dos recursos seguirá instrumento já existente na área educacional, neste caso, o repasse dos recursos orçamentários e financeiros será disponibilizado pelo MEC por meio de Termo de Cooperação para a descentralização de créditos.
Segundo enunciado no caput do Art. 11, da portaria interministerial $n^{0} 18$, de 18 de dezembro de 2013, "As despesas decorrentes deste instrumento correrão à conta das dotações orçamentárias anualmente consignadas ao MEC, observados os limites estipulados pelo Poder Executivo na forma da legislação orçamentária e financeira".

Apesar do SNC e PNC trabalharem com a articulação entre os entes federativos, e mesmo considerando que o MinC dialoga e se articula com instituições de ensino superior no geral, a iniciativa do PMCU se restringe, na atualidade, às IFES apenas, não considerando ainda, neste primeiro edital, o beneficio direto às Instituições de Ensino Superior Estaduais e Municipais. Esta questão foi muito debatida e criticada, tanto no Seminário Cultura e Universidade quanto no I Encontro Nacional do Ensino Superior das Artes, segundo Juana Nunes, atual Secretária de Educação e Formação Artístico Cultural do MinC, entrevistada ano passado quando gestora da Diretoria de Educação e Comunicação para a Cultura da SPC/MinC.

A Secretária também esclarece que, para que o lançamento do programa não sofresse mais atrasos, definiu-se que a principio seriam contempladas apenas a esfera Federal do ensino superior. Sendo que, a opção pelo atendimento exclusivo às IFES se deu pelas facilidades dos procedimentos burocráticos da modalidade de descentralização de recursos do MEC.

Esta é uma questão que constitui certa contradição em consideração aos princípios do SNC e evidencia o desafio da institucionalização das políticas culturais, resvalando na discussão sobre o Federalismo prático das políticas de cultura, visto que a complexidade do Federalismo se dá a partir da impossibilidade dos instrumentos legais da federação se subordinarem à estrutura constitucional da União, que centraliza e muita vez enrijece os métodos de aplicação das políticas (CUNHA; RIBEIRO, 2013). 
Ainda assim, as universidades estaduais e municipais podem participar apenas por meio de parcerias com as IFES. Vale destacar que a parceria também pode ser feita com qualquer instituição educacional e cultural, criando redes de articulação, conforme enumerado nos parágrafos $1^{\circ}$ e $2^{\circ}$ do Art. $1^{\circ}$, da portaria interministerial $n^{0} 18$, de 18 de dezembro de 2013. O que não é possível ser viabilizado pelo programa, no momento, é o envio direto dos recursos para as universidades estaduais e municipais, devido à legislação que regulamenta o repasse direto apenas na esfera federal.

Sendo assim, para que houvesse a inclusão das universidades estaduais e municipais, a movimentação orçamentária necessariamente ocorreria por convênio, gerando a obrigatoriedade de contrapartida e outros procedimentos burocráticos para os quais o MinC não tem condições de se responsabilizar no momento, pois demandaria maior infraestrutura de servidores qualificados para operacionalizar tais procedimentos. Espera-se que esta questão seja superada ao longo do processo de continuidade previsto na portaria do programa.

0 recorte para o gerenciamento desta verba fica claro no Art. $8^{\circ}$, da portaria interministerial $n^{0} 18$, de 18 de dezembro de 2013:

Art. $8^{\circ}$ - Para o atendimento dos Planos de Cultura aprovados pelo Comitê Técnico serão utilizados instrumentos próprios já utilizados pelo MEC que fixarão os recursos financeiros destinados às instituições selecionadas dentre as relacionadas no $\S 1^{\circ}$ do art. $1^{\circ}$ selecionadas, vinculando os repasses ao cumprimento das etapas.

Parágrafo único - 0 atendimento dos Planos de Cultura é condicionado à capacidade orçamentária e operacional do MEC.

A intenção, desde os primeiros momentos da estruturação do PMCU, foi estimular o pensamento crítico sobre o papel das políticas culturais no ensino superiore, inclusive, prospectando antecipações sobre o impacto de sua abrangência. Nesta perspectiva, a principal indução pretendida era a necessidade de que as instituições acadêmicas organizassem, dentro de seu plano político pedagógico, um Plano de Cultura para a Universidade (PCU). Sendo assim, o primeiro edital teve, como requisito obrigatório, a elaboração de plano de cultura com período de 12 a 24 meses.

Os Planos de Cultura inscritos tiveram o desafio de articular e promover a interface de diálogo entre educação, arte e cultura, estabelecendo objetivos, ações e metas, considerando sua relação com as manifestações, expressões, produções artísticas e culturais e seu território. Conforme enunciado no art. $5^{\circ}$ da referida portaria.

Com esta perspectiva, o MinC procurou induzir, nas universidades proponentes, a construção de um plano de ações culturais, na expectativa da emergência de um processo de elaboração de um plano mais processual e com uma temporalidade maior, e não apenas de alguns meses. Sendo assim, as universidades podem fazer isso de forma mais aprofundada e consolidada, envolvendo posteriormente, em uma elaboração mais sólida, a participação social dos agentes no âmbito da extensão, da produção cultural estudantil e docente, das comunidades e também a oportunidade de se pensar os cursos de Formação em Artes, além de promover a circulação da produção de pesquisas existentes na academia, sendo este um espaço privilegiado para fazer fluir este diálogo.

Mesmo com as limitações burocráticas, esperase que a política se equalize com o horizonte político e institucional que vem sendo instituído com a implementação do SNC, criando alternativas que sejam convergentes e dialoguem com o objetivo maior, que é a universalização dos bens e serviços culturais. A grande expectativa é que esta política se estabeleça como política de Estado e não apenas de governo, consolidando o estreitamento de ações entre o MinC e o MEC.

Importa ressaltar que, pela primeira vez o governo federal promove a descentralização de recursos para 
investimento em uma agenda contínua, que fomente a transversalidade das políticas culturais e de educação para o ensino superior do país e a reflexão e reivindicação de aprimoramento das políticas desta natureza, de forma a assegurar os insumos para além das perspectivas consonantes à gestão pública dos governos subsequentes.

\section{PROPOSIÇ̃̃ES PARA O DESENVOLVIMENTO DO PROGRAMA MAIS CULTURA NAS UNIVERSIDADES E CONSIDERAÇÕES FINAIS}

Destacamos aqui análises propositivas mais específicas acerca da gestão do PMCU e dos possíveis impactos deste programa no cenário das políticas públicas para o ensino superior do país, de forma a oferecer pontuações que impliquem em ações que possam contribuir para o aprimoramento do próprio programa. Sem a pretensão de gerar um diagnóstico hipotético, mas no intuito de elucidar pontos que sinalizam fragilidades, apresentamos a seguir algumas sugestões.

A partir da condição apresentada acerca da instrumentalização dos recursos destinados para os possíveis beneficiados pelo PMCU nesta primeira etapa de sua implantação, que se restringem apenas às IFES, não contemplando diretamente as das esferas Estaduais e Municipais, algumas reflexões de caráter propositivo podem ser tecidas. Considerando que tal situação é dissonante às diretrizes gerais do SNC, a questão apontada se mostra como limitadora. A quinta diretriz, apontada no art. 4 da referida portaria “articular a educação superior e a educação profissional e tecnológica com a educação básica, visando ao seu fortalecimento, qualificação e a sua articulação no território”, evidencia a necessidade lógica de maior abrangência dos beneficiados com o programa, obedecendo o pacto federativo.
Como a demanda de inclusão das instituições de ensino superior das esferas estaduais e municipais foi fortemente debatida nos ambientes de concepção do PMCU, este advento evidencia o ponto mais desafiante, no momento, para 0 desenvolvimento do programa. Apesar de o MinC dialogar numa perspectiva inclusiva para todas as esferas federativas, com o SNC e PNC, promovendo a integração entre tais entes federativos, a iniciativa do PMCU ainda não contempla amplamente tais metas, sendo reconhecido pelo MinC a necessidade de considerar esta prerrogativa e, de amadurecer com o MEC um diálogo para esta questão.

Propõem-se aqui também a criação de uma secretaria ou diretoria específica da cultura dentro da estrutura organizacional do MEC, de forma a tratar com competência a complexidade da dimensão cultural das políticas educacionais. Em médio prazo, estima-se também a necessidade de uma expansão qualificada do quadro de funcionários que participam o Comitê Técnico Gestor do Programa, podendo, assim, operacionalizar melhor os processos e etapas do PMCU. Cientes de que este desafio demanda maior interesse político e recursos para sua estruturação, além de que esta é uma pauta que tangencia todas as esferas governamentais do país.

Também, poderiam ser definidas novas modalidades de fomento e repasse de recurso a exemplo do Programa Dinheiro Direto na Escola (PDDE), que prevê o repasse direto de recursos para todas as instituições públicas da rede básica de ensino. Sendo a escola uma unidade executora, ela tem uma conta e recebe o repasse direto do MEC, sem restrições. Logo, se o MEC consegue ter um instrumento que viabiliza o repasse direto de recursos para escolas públicas, esse modelo de programa pode ser expandido para o ensino superior, englobando todas as universidades públicas por meio de um decreto presidencial, com previsão legal na Lei $n^{0}$ 9.394 de 1996 que estabelece as diretrizes e bases da educação, assim como foi o do PDDE. 
0 debate sobre esta questão, que transversaliza com o das alianças federativas e os pactos que podem e devem ser efetivamente realizados, já vem sendo apontado como um dos desafios urgentes. A UNESCO, em sua publicação Desafios e Perspectivas da Educação Superior Brasileira para a Próxima Década, aponta sugestões neste sentido:

Alternativa viável para a expansão qualificada da escola superior diz respeito ao fortalecimento do regime de colaboração entre a União e os entes federados (estados, municípios e Distrito Federal), a exemplo do que já acontece com a educação básica por meio do plano de metas Compromisso Todos pela Educação, instituído pelo Decreto 6.094/2007. Ou seja, se existe uma parceria do governo federal com as secretarias municipais e estaduais de educação em prol do desenvolvimento da educação básica, caberia também uma parceria maior com as IES públicas municipais e estaduais. (UNESCO, 2012, p. 158)

Em curto e médio prazo, outras formas de articulação que potencializem o programa podem ser instauradas. É necessário que haja, para além do instituído Fórum Nacional de Formação e Inovação em Arte e Cultura, a prospecção do "objetivo de articular e congregar as instituições relacionadas", explícito no caput do art. $2^{\circ}$ da referida portaria, podendo ser efetivada, também, pelo estabelecimento de redes articuladas com a sociedade civil, para que se amplie o seu espaço de fala, não apenas como público-alvo, mas como parceiro estratégico.

Fica evidente, com o crescimento do ensino superior no país, a necessidade de efetivação e maturação de políticas públicas nas universidades e faculdades, de forma a garantir uma relação próxima e orgânica com a sociedade civil. Há muito ainda o que se discutir e amadurecer no que se refere ao novo papel das universidades publicas junto ao MinC e ao MEC, de forma a garantir as articulações estratégicas para 0 aprimoramento das respectivas políticas públicas.

A adesão de 98 das 101 Instituições Federais de Ensino Superior ao edital do Programa (BRASIL,
2015), já se constitui como um indicador muito positivo. Entretanto, espera-se que os PCUs possam contribuir para a consolidação das universidades como ambientes de exercício criativo/artístico, de formação e de diálogo por meio e com a cultura e a sociedade como um todo.

\section{REFERÊNCIAS}

BARBALHO, Alexandre; RUBIM, Antônio Albino Canelas (Org.). Políticas culturais no Brasil. SalvadorBA: EDUFBA, 2007.

BARBALHO, Alexandre; BARROS, José Márcio; CABRALE, Lia (Org.). Federalismo e políticas

culturais no Brasil. Salvador-BA: EDUFBA, 2013

BARROS, José Marcio. Diversidade cultural e gestão: apontamentos preliminares. Texto apresentado no IV ENECULT. Promovido pelo Centro de Estudos Multidisciplinares em Cultura da UFBA em 2008.

\section{BARROS, José Marcio. Seminário Cultura e}

Universidade na Mesa: A formação de gestores culturais e o Sistema Nacional de Cultura. Promovido pelo MINC e UFBA, 2013

BOTELHO, Isaura. Cultura e Universidade: Reconstituindo as Trajetórias dos Diálogos Institucionais. Políticas Culturais: Teoria e Práxis. Observatório Itaú Cultural, 2011

\section{BRASIL. Constituição da República Federativa do}

Brasil. Artigos 205, 206, 207, 208, 210, 214, 215, 216 e 216-A, 1988.

BRASIL. Lei no 10.172, de 10 de janeiro de 2001. Institui o Plano Nacional de Educação, 2001.

BRASIL. Ministério da Educação e Cultura (MEC). Plano de Desenvolvimento da Educação (PDE). Brasília: MEC, 2007. 
BRASIL. Decreto no 6.096, de 24 de abril de 2007. Institui o Programa de Apoio a Planos de Reestruturação e Expansão das Universidades Federais - Reuni, 2007.

BRASIL. Programa Mais Cultura nas Universidades tem quase 100\% de adesão. 6 maio 2015. Dia da cultura. Disponível em: <http://goo.gl/0T2q35> Acesso em: 3 jul. 2015.

\section{CARTA DE OURO PRETO, 2014.}

\section{MINISTÉRIO DA CULTURA. Oficinas do Sistema} Nacional de Cultura / Organização: Lia Calabre. Brasília: Ministério da Cultura, 2006. p.144.

\section{MINISTÉRIO DA CULTURA. Plano Nacional de}

Cultura: Diretrizes Gerais. 2.ed. Brasília: Ministério da Cultura, 2008.

\section{MINISTÉRIO DA CULTURA. Cultura em três}

dimensões: As políticas do Ministério da Cultura de 2003 a 2010. Brasília: MinC, 2011.

\section{MINISTÉRIO DA CULTURA. Revista $1^{\circ}$ Seminário}

Cultura e Universidade: Bases para uma Política
Nacional de Cultural para as Instituições de Ensino Superior. MinC/UFBA. 2014.

MORIN, Edgar. Os sete saberes necessários à educação do futuro. 3.ed. São Paulo: Cortez; BrasíliaDF: UNESCO, 2001.

\section{NASCIMENTO, Alberto F. Política cultural e} financiamento do setor cultural. Encontro de estudos multidisciplinares em cultura. Salvador: Edufba, maio 2008.

\section{RUBIM, Antonio Albino Canelas. Políticas culturais} no Brasil: Desafios contemporâneos. S/d.

\section{UNESCO. Declaração Mundial sobre Educação Superior no Século XXI. Piracicaba: UNIMEP, 1998.}

\section{UNESCO. Tendências da educação superior para o século XXI. Brasília: Unesco/CRUB, 1999.}

\section{UNESCO/CNE/MEC. Desafios e perspectivas da} educação superior brasileira para a próxima década. SPELLER, Paulo; ROBL; Fabiane; MENEGHEL, Stela Maria. (Org.). Brasília: 2012. 164p.

3. Analista Técnico-Administrativo do Ministério da Cultura, lotado na Secretaria de Articulação Institucional, Graduado em Relações Internacionais pela UnB; Especialista em gestão cultural - UFBA; Mestrando do Programa Multidisciplinar de Pós-Graduação em Cultura e Sociedade - IHAC/UFBA. E-mail: tony.bezerra@cultura.gov.br 